\title{
Development of operando Techniques for Battery Study using SPICA \\ Masao Yonemura ${ }^{1}$ \\ ${ }^{1}$ High Energy Accelerator Research Organization (KEK), Tokai, Japan \\ E-mail: yone@post.kek.jp
}

SPICA, a new special environment powder neutron diffractometer was built at BL09 in the Material and Life science Facility (MLF) of the Japan Proton Accelerator Research Complex (J-PARC). This is the first instrument dedicated for the study of next-generation batteries and is optimized for real-time measurements to determine the structural changes of battery materials at the atomic level. To observe real-time structural changes of materials in the practical device, the diffractometer with a good balance between intensity and resolution is needed. Therefore, SPICA is designed so that the sample position locates at $52 \mathrm{~m}$ from the thin side of the decoupled-poisoned moderator for high resolution as well as the elliptical supermirror guide tube is adopted to enhance neutrons at the sample position for high intensity. As the results, the highest resolution, $\Delta \mathrm{d} / \mathrm{d}$ of $0.09 \%$ is achieved at the back-scattering bank, and the neutron intensity gains 4 times larger than the tapered-straight guide [1].

The operando diffraction experiments were carried out. The structural changes of the active materials, which is dependent on the lithium content, is clearly observed [2]. The lattice parameters for the anode and cathode materials as a function of the lithium content were extracted from the diffraction patterns. And an automatic data analysis procedure was also developed. Z-Rietveld which is an original Rietveld refinement software in J-PARC [3] can handle the large number of diffraction data provided by the operando observation and sequentially refine the structures of both electrodes. The fullscale usage of SPICA has just begun. In this presentation, the more details of operando measurements on SPICA will be reported.

Acknowledgement

This work was supported by the Research and Development Initiative for Scientific Innovation of New Generation Batteries (RISING) project of the New Energy and Industrial Technology Development Organization (NEDO). This work was carried out as the S-type project of KEK (Proposal No. 2009S10, 2014S10). The author wishes to make grateful acknowledgement to my collaborator, (KEK) T. Kamiyama, Y. Ishikawa, S. Torii, (Kyoto Univ.) K. Mori, T. Fukunaga, Y. Onodera, T. Naka, M. Morishima, M. Shiotani, T. Matsunaga, H. Komatsu, Y. Ukyo, H. Arai, Y. Uchimoto, Z. Ogumi, (Yamagata Univ.) R. Tomiyasu, (Natl. Inst. Adv. Ind. Sci. and Tech.) K. Kino, (Tokyo Inst. Tech.) S. Taminato, M. Hirayama, R. Kanno, (Tokyo Univ. of Sci.) N. Ishida, N. Kitamura, Y. Idemoto.

[1] Yonemura, M. et al. J. Phys. Conf. Ser. (2014), 502 article number 012053.

[2] Taminato, S. et al. (2016), Scientific Reports 6 article number 28843.

[3] Oishi, R. et al. (2009) Nucl. Instrum. Methods Phys. Res. Sect. A, 600(1) 94-96.

Keywords: neutron, oprando, battery 